\title{
Communication
}

\section{Consumption of Sports and Energy Drinks by High School Athletes in the United States: A Pilot Study}

\section{Sarah K. Fields ${ }^{1, *}$, James MacDonald ${ }^{2, \dagger}$, Allan M. Joseph ${ }^{3, \dagger}$, Loren E. Wold ${ }^{4, \dagger}$, Christy L. Collins ${ }^{5, \dagger}$ and R. Dawn Comstock ${ }^{6, \uparrow}$ \\ 1 Department of Communication, University of Colorado Denver, Campus Box 176, P.O. Box 173364, Denver, CO 80217-3364, USA}

2 Division of Sports Medicine, Department of Pediatrics, Nationwide Children's Hospital, One Children's Drive, Columbus, $\mathrm{OH} 43205$, USA;

E-Mail: James.MacDonald@nationwidechildrens.org

3 Warren Alpert Medical School, Brown University, Box G-9999, Providence, RI 02915, USA; E-Mail: allan_joseph@brown.edu

4 Colleges of Nursing and Medicine, The Ohio State University, 1585 Neil Ave., Columbus, OH 43210, USA; E-Mail: wold.5@osu.edu

5 OhioHealth Research \& Innovations Institute, Riverside Methodist Hospital, 3545 Olentangy River Road, Columbus, OH 43234, USA; E-Mail: Christy.Collins@ohiohealth.com

6 Department of Epidemiology, Colorado School of Public Health, 13001 E. 17th Place, Campus Box B119, Aurora, CO 80045, USA; E-Mail: Dawn.Comstock@ucdenver.edu

$\dagger$ These authors contributed equally to this work.

* Author to whom correspondence should be addressed; E-Mail: Sarah.Fields@ucdenver.edu; Tel.: +1-303-315-1919; Fax: +1-303-315-1920.

Academic Editor: Juan Del Coso Garrigós

Received: 5 August 2015 / Accepted: 15 September 2015 / Published: 22 September 2015

\begin{abstract}
Sports and energy (S/E) drinks are commonly used by high school (HS) athletes, yet little is known about this population's consumption patterns or the drinks' side-effects. The objectives of this pilot study were to survey HS athletes about their use of S/E drinks and assess potential side-effects. One hundred American HS athletes (72 were female; 27 were male; one did not identify gender) were part of a cross-sectional internet-based survey. The mean age of the athletes was $16.0 \pm 1.1$ years. The athletes self-reported S/E consumption patterns, motivations for consumption, and drink side-effects. Nearly two-thirds $(59.5 \%)$ of athletes surveyed were at least occasional users of sports drinks, and more than
\end{abstract}


one-third $(37.3 \%)$ were at least occasional users of energy drinks. Of the athletes who had ever drunk an S/E drink, $49.5 \%$ drank their first sport drink at $\leq 8$ years and $41.3 \%$ consumed their first energy drink $\leq 11-12$ years of age. The most common motivation for consumption of sports drinks was to rehydrate $(84.1 \%)$ and of energy drinks was to gain energy (61.8\%). Side effects of S/E drinks were frequently reported; $25.3 \%$ of energy drink users reporting being nervous/jittery after consumption. Thus HS athletes should be cautioned about consumption of $\mathrm{S} / \mathrm{E}$ drinks until more is understood about their short- and long-term side-effects.

Keywords: BMI; caffeine; obesity; energy drink; sports drink; high school; athletes

\section{Introduction}

Approximately 8 million United States (US) high school (HS) students participated in sports in 2013-2014 [1]. Though research has analyzed consumption of high-calorie drinks by HS students [2], sports and energy (S/E) drink consumption by HS athletes has largely remained unstudied. The literature lacks data regarding prevalence and patterns of $\mathrm{S} / \mathrm{E}$ drink consumption and the drinks' possible side-effects in this specific population.

Sports and energy drinks are distinctly different. Sports drinks aim primarily to replace water and salts lost through perspiration; they contain water, electrolytes, and carbohydrates [3]. Energy drinks have caffeine as their main active ingredient [4]. Energy drinks may also contain sugars, artificial sweeteners, vitamins, taurine, ginseng, and L-carnitine [4]. Specific ingredients in individual S/E drinks may vary widely.

Some negative effects of $\mathrm{S} / \mathrm{E}$ drink ingredients have been investigated. There is a strong link between consumption of sweetened drinks and childhood obesity [5]. Sports drinks can harm teeth [6]. Energy drinks may have additional psychological effects, such as anxiety and insomnia caused by the high quantities of caffeine [7]. Teenagers who consume energy drinks are more likely to use alcohol and drugs [8]. Energy drinks themselves can result in acute cardiovascular issues and possible chronic concerns [9].

The consumption patterns of S/E drinks by HS athletes, and the drinks' effects on this group, are not fully known. The objectives of this pilot study were to survey HS athletes about their use of S/E drinks and assess potential side-effects. Our specific aims were to: (1) describe S/E drink consumption prevalence and patterns; (2) assess self-reported side-effects; and (3) determine an association between $\mathrm{S} / \mathrm{E}$ drink consumption and body mass index (BMI).

\section{Methods}

An internet-based survey was administered to a convenience sample of 100 athletes from schools participating in the National High School Sports-Related Injury Surveillance Study (High School $\mathrm{RIO}^{\mathrm{TM}}$ ). This national ongoing prospective surveillance study has been described previously $[10,11]$. The authors e-mailed Certified Athletic Trainers (ATs) at participating schools in May 2009 and 
explained the study objectives and procedures. The ATs were to inform their schools' athletes about the study and provide an internet link to the survey. Any athlete from a High School RIO ${ }^{\text {TM }}$ study school with internet access and English literacy was eligible to participate. The first 100 students completing the survey received a $\$ 5$ gift certificate; those surveys were used for data analysis. The study was approved by the Institutional Review Board at Nationwide Children's Hospital.

The anonymous survey captured self-reported variables including demographics, athletic participation, patterns of consumption, height and weight, and side-effects of S/E drinks. Student athletes were provided a drop down list of specific types of drinks in each category (i.e., sports and energy) as well as the ability to write in additional answers not included in the drop down options. Similarly, they were provided a drop down list of types of potential side-effects as well as the ability to write in additional answers not included in the drop down options. Consumption frequency was classified into seven categories based on number of days student athletes consumed $\mathrm{S} / \mathrm{E}$ drinks per month: non-users, rare users (1-2 days per month), occasional users (3-5 days), frequent users (6-9 days), regular users (10-19 days), heavy users (20-29 days), and habitual users (every day). Age- and gender-specific BMI percentiles were calculated using guidelines developed by the Centers for Disease Control and Prevention [12].

Data were analyzed using SPSS (version 14.0; SPSS Inc., Chicago, IL, USA) and Epi Info (version 6.0; CDC, Atlanta, GA, USA). Odds ratios (ORs) were calculated to determine strengths of association. The chi-square test and $95 \%$ confidence intervals (CIs) were used to evaluate statistical significance. CIs not containing 1.0 and $p<0.05$ were considered significant.

\section{Results and Discussion}

Demographic data are reported in Table 1. More females $(n=72)$ than males $(n=27)$ participated. Respondents most commonly participated in track \& field, soccer, and basketball.

Table 1. Demographic Characteristics of High School (HS) Athletes Completing Survey.

\begin{tabular}{cccc}
\hline & Female $(\boldsymbol{n}=\mathbf{7 2})$ & Male $(\boldsymbol{n}=\mathbf{2 7})$ & Total $(\boldsymbol{n}=\mathbf{1 0 0}) *$ \\
\hline \multicolumn{2}{c}{ Year in School } \\
\hline Freshman & $23.6 \%$ & $0.0 \%$ & $17.0 \%$ \\
Sophomore & $19.4 \%$ & $0.0 \%$ & $14.0 \%$ \\
Junior & $36.1 \%$ & $77.8 \%$ & $47.0 \%$ \\
Senior & $20.8 \%$ & $22.2 \%$ & $22.0 \%$ \\
Total & $100.0 \%$ & $100.0 \%$ & $100.0 \%$ \\
\hline \multicolumn{5}{c}{ Age (years) } \\
Minimum & 12 or younger & 16 & 12 or younger \\
Maximum & 18 or older & 18 or older & 18 or older \\
Mean (St. Dev) $* *$ & $16(1.2)$ & $17(0.6)$ & $16(1.1)$ \\
\hline \multicolumn{5}{c}{ BMI } \\
Minimum & 17.2 & 18.2 & 17.2 \\
Maximum & 33.3 & 30.0 & 33.3 \\
Mean (St. Dev) & $22.6(3.4)$ & $23.0(2.6)$ & $22.7(3.2)$ \\
\hline
\end{tabular}


Table 1. Cont.

\begin{tabular}{cccc}
\hline & Female $(\boldsymbol{n}=\mathbf{7 2})$ & Male $(\boldsymbol{n}=\mathbf{2 7})$ & Total $(\boldsymbol{n}=\mathbf{1 0 0}) *$ \\
\hline \multicolumn{2}{c}{ Sports Played } \\
\hline Track \& Field & $19.4 \%$ & $11.1 \%$ & $17.0 \%$ \\
Soccer & $12.5 \%$ & $25.9 \%$ & $17.0 \%$ \\
Basketball & $9.7 \%$ & $37.0 \%$ & $17.0 \%$ \\
Field Hockey & $16.7 \%$ & $0.0 \%$ & $12.0 \%$ \\
Lacrosse & $12.5 \%$ & $7.4 \%$ & $11.0 \%$ \\
Football & $2.8 \%$ & $29.6 \%$ & $10.0 \%$ \\
\hline
\end{tabular}

* One student failed to report their gender; ** Students who responded as "12 or younger" or "18 or older" were counted as 12 years old and 18 years old, respectively when calculating the mean; ${ }^{\dagger}$ Top six sports in survey are listed; respondents were able to report multiple sports played.

The most popular S/E drinks consumed are listed in Table 2. Sports drinks were more commonly consumed than energy drinks.

Table 2. Five Most Popular Sports Drinks and Energy Drinks Consumed by HS Athletes Completing the Survey. ${ }^{\dagger}$

\begin{tabular}{cccc}
\hline & Female $(\boldsymbol{n}=\mathbf{7 2})$ & Male $(\boldsymbol{n}=\mathbf{2 7})$ & Total $(\boldsymbol{n}=\mathbf{1 0 0}) *$ \\
\hline \multicolumn{3}{c}{ Sports Drink } \\
\hline Gatorade & $98.6 \%$ & $100.0 \%$ & $99.0 \%$ \\
Powerade & $91.5 \%$ & $96.3 \%$ & $92.9 \%$ \\
Muscle Milk & $2.9 \%$ & $51.9 \%$ & $16.5 \%$ \\
Promax & $5.6 \%$ & $14.8 \%$ & $8.0 \%$ \\
Myoplex & $4.2 \%$ & $11.1 \%$ & $6.1 \%$ \\
\hline \multicolumn{5}{c}{ Energy Drink } \\
Red Bull & $62.0 \%$ & $85.2 \%$ & $68.7 \%$ \\
Monster & $50.0 \%$ & $70.4 \%$ & $56.0 \%$ \\
Amp & $46.5 \%$ & $66.7 \%$ & $52.5 \%$ \\
Rockstar & $47.2 \%$ & $63.0 \%$ & $52.0 \%$ \\
Full Throttle & $26.8 \%$ & $40.7 \%$ & $30.3 \%$ \\
\hline
\end{tabular}

Data based on drinks students have ever tried; * One student failed to report their gender; Multiple selections are allowed so column totals $>100 \%$.

Consumption frequency was classified into 7 categories based on number of drinks consumed per month. For sports drinks, $13.8 \%$ of students were non-users (consumed a sports drink 0 days per month), $26.6 \%$ rare users (consumed a sports drink 1 or 2 days per month), 23.4\% occasional users (consumed a sports drink 3 to 5 days per month), 13.8\% frequent users (consumed a sports drink 6 to 9 days per month), 10.6\% regular users (consumed a sports drink 10 to 19 days per month), 7.4\% heavy users (consumed a sports drink 20 to 29 days per month), and $4.3 \%$ habitual users (consumed a sports drink every day of the month). Energy drink consumption was less common: $30.7 \%$ of students were non-users, $32.0 \%$ rare users, $20.0 \%$ occasional users, $12.0 \%$ frequent users, $1.3 \%$ regular users, $2.7 \%$ heavy users, and $1.3 \%$ habitual users. Although there were no significant differences in consumption 
prevalence between males and females, a higher proportion of males consumed sports drinks more frequently than females.

Of athletes who ever drank a sports drink, $49.5 \%$ consumed their first at $\leq 8$ years, $19.2 \%$ at $9-10$, $16.2 \%$ at $11-12,7.1 \%$ at $13-14$, and $8.1 \%$ at $15-16$. Energy drink consumption generally occurred later, with $12.5 \%$ consuming their first at $\leq 8$ years, $11.3 \%$ at $9-10,17.5 \%$ at $11-12,27.5 \%$ at $13-14$, $27.5 \%$ at $15-16$, and $3.8 \%$ at $\geq 17$. Overall, $41.3 \%$ consumed their first energy drink $\leq 11-12$ years of age.

Athletes consumed sports drinks before (41.1\%), during (59.3\%), and after (67.0\%) sports; at meals (16.5\%); and at "other times" (36.3\%) to rehydrate (84.1\%), for taste $(79.3 \%)$, and because of parental purchasing (33.7\%). Less than a fifth $(18.0 \%)$ consumed them to improve athletic performance. Others' influence also motivated consumption; $11.2 \%$ consumed them because teammates did and $10.1 \%$ under coaches' orders. Demonstrating a lack of knowledge regarding sports drinks' effects, 31.5\% consumed them for energy and $16.9 \%$ to stay awake.

Athletes consumed energy drinks before (17.2\%), during (14.1\%), and after (18.5\%) sports; at meals $(6.2 \%)$; and at "other times" $(67.7 \%)$ to gain energy $(61.8 \%)$, for taste $(60.3 \%)$, to stay awake (57.4\%), to rehydrate (29.4\%), to improve decision making (13.2\%), and because teammates did (13.4\%). Males in particular drank them to gain energy $(80.0 \%)$ and improve athletic performance $(20.0 \%)$.

Self-reported side effects were frequent (Table 3). Over a quarter of energy drink users reported feeling nervous/jittery after consumption. Increased frequency of consuming either type of drink was not significantly associated with the likelihood of experiencing a side effect.

Table 3. Self-reported Side-effects of Sports Drinks and Energy Drinks. ${ }^{\dagger}$

\begin{tabular}{cccc}
\hline & Female $(\boldsymbol{n}=\mathbf{7 2})$ & Male $(\boldsymbol{n}=\mathbf{2 7})$ & Total $(\boldsymbol{n}=\mathbf{1 0 0})$ \\
\hline & Sports Drinks & & \\
\hline Dizzy, lightheaded & $6.9 \%$ & $0.0 \%$ & $5.1 \%$ \\
Nausea & $11.1 \%$ & $0.0 \%$ & $8.1 \%$ \\
Blurred vision & $0.0 \%$ & $0.0 \%$ & $0.0 \%$ \\
Dehydrated & $15.3 \%$ & $7.4 \%$ & $13.1 \%$ \\
Abdominal cramping/diarrhea & $9.7 \%$ & $3.7 \%$ & $8.1 \%$ \\
Nervous/jittery & $12.5 \%$ & $3.7 \%$ & $10.1 \%$ \\
Insomnia & $6.9 \%$ & $3.7 \%$ & $6.1 \%$ \\
Other ** & $2.8 \%$ & $0.0 \%$ & $2.0 \%$ \\
\hline Dizzy, lightheaded & Energy Drinks & & \\
Nausea & $13.9 \%$ & $14.8 \%$ & $14.1 \%$ \\
Blurred vision & $12.5 \%$ & $7.4 \%$ & $11.1 \%$ \\
Dehydrated & $2.8 \%$ & $0.0 \%$ & $2.0 \%$ \\
Abdominal cramping/diarrhea & $15.3 \%$ & $7.4 \%$ & $5.1 \%$ \\
Nervous/jittery & $5.6 \%$ & $3.7 \%$ & $25.3 \%$ \\
Insomnia & $26.4 \%$ & $22.2 \%$ & $9.1 \%$ \\
Other*** & $11.1 \%$ & $3.7 \%$ & $5.1 \%$ \\
\hline
\end{tabular}

$\dagger$ Data based on those who consume S/E drinks, total percentages are therefore $<100 \%$; One student failed to report their gender; ** "Other" for sports drinks included dry mouth and feeling "hyper"; *** "Other" for energy drinks included feeling "hyper", addiction, heart palpitations, anxious, and increased heart rate; Students were allowed to choose multiple side effects 


\section{Conclusions}

This pilot study surveyed HS athletes from across the United States to gather information about consumption patterns and motivation for consumption of $\mathrm{S} / \mathrm{E}$ drinks and to explore the side-effects of $\mathrm{S} / \mathrm{E}$ drinks. S/E drinks were consumed frequently in this population. We also found that of the athletes who had consumed at least one S/E drink, their exposure to the product often came early.

Side-effects were reported frequently with $13.1 \%$ of all sports drink users reporting dehydration as a side-effect and $25.3 \%$ of all energy drink users reporting being nervous/jittery. The only side-effect that no young athletes reported having after consuming sports drinks was blurred vision. Given that every side-effect, except blurred vision in sports drink consumers, was reported by at least some S/E consumers, our study indicates this is a matter of concern. Although our study sheds initial light on the potential side-effects of S/E drink consumption, given the growing popularity of S/E drinks and the trend toward younger children's consumption [7], further research on their short and long-term health effects in young athletes is needed.

Our findings should be interpreted with caution given the study limitations. For example, this pilot study was conducted in a convenience sample of 100 student athletes. This limit, imposed by the small amount of funds available for participant incentives, raises concerns regarding generalizability to the broader student athlete population. Additionally, there was a preponderance of female subjects in this study, yet prior research indicates S/E drinks are typically used more frequently by males [7]. Football players were underrepresented in our sample as football is the most frequently played HS sport in the U.S. [1]. Finally, all data were self-reported, opening the possibility of reporting biases. Despite these limitations, this work appears to be one of the first to discuss the age of first consumption of S/E drinks, motivation for consumption, and side effects. While future research should confirm this work, our findings should drive hypotheses for further work in this area.

The young age of first consumption of $\mathrm{S} / \mathrm{E}$ drinks in this population is concerning as were some of the motivations for consumption. Additionally, it appears negative side effects, such as being nervous/jittery, are common among young athletes consuming energy drinks. Additional research into this area is needed, including larger surveys with increased power. HS athletes should currently be cautioned regarding consumption of S/E drinks until such research better elucidates the effects of these drinks in this population.

\section{Acknowledgments}

The author A.M.J. gratefully acknowledges the Glynn Family Honors Program Grant and Center for Undergraduate Scholarly Engagement Grant from the University of Notre Dame for his financial stipend support during the writing of this project.

\section{Author Contributions}

Authors contributed equally to effort and content of the manuscript.

\section{Conflicts of Interest}

The authors declare no conflict of interest. 


\section{References}

1. National Federation of State High School Athletic Associations (NFHS). 2013-14 High School Athletics Participation Survey. Available online: http://www.nfhs.org/ParticipationStatics/PDF/ 2013-14_Participation_Survey_PDF.pdf (accessed on 31 July 2015).

2. Robert Wood Johnson Foundation Statement Regarding Evaluation of School Beverage Guidelines. Available online: http:/www.rwjf.org/en/about-rwjf/newsroom/newsroomcontent/2010/03/robert-wood-johnson-foundation-statement-regarding-evaluation-of.html (accessed on 1 August 2015).

3. MacDonald, J. The potential adverse health effects of energy drinks. Am. Fam. Physician 2013, $87,321$.

4. Seifert, S.M.; Schaechter J.L.; Hershorin E.R.; Lipshultz, S.E. Health effects of energy drinks on children, adolescents, and young adults. Pediatrics 2011, 127, 511-528.

5. Ludwig, D.S.; Peterson, K.E.; Gortmaker, S.L. Relation between consumption of sugar-sweetened drinks and childhood obesity: a prospective, observational analysis. Lancet 2001, 357, 505-508.

6. Milosevic, A. Sports drinks a hazard to teeth. Br. J. Sports Med. 1997, 31, 28-30.

7. Heckman, M.A.; Sherry, K.; Gonzalez de Mejia, E. Energy drinks: An assessment of their market size, consumer demographics, ingredient profile, functionality, and regulations in the United States. Comp. Rev. Food Sci. Food Saf. 2010, 9, 303-317.

8. Terry-McElrath, Y.M.; O’Malley, P.M.; Johnston, L.D. Energy drinks, soft drinks, and substance use among United States secondary school students. J. Addict. Med. 2014, 8, 6-13.

9. Higgins, J.P.; Yarlagadda, S.; Yang, B. Cardiovascular complications of energy drinks. Beverages 2015, 1, 104-126.

10. Rechel, J.A.; Yard, E.E.; Comstock, R.D. An epidemiologic comparison of high school sports injuries sustained in practice and competition. J. Athl. Train. 2008, 43, 197-204.

11. Sports-Related Injuries among High School Athletes-U.S., 2005-06 School Year. Available online: http://www.cdc.gov/mmwr/PDF/wk/mm5538.pdf (accessed on 17 September 2015).

12. Centers for Disease Control and Prevention, Division of Nutrition, Physical Activity, and Obesity: About Child and Teen BMI. Available online: http://www.cdc.gov/healthyweight/assessing/bmi/ childrens_bmi/about_childrens_bmi.html\#HowIsBMICalculated (accessed on 1 August 2015).

(C) 2015 by the authors; licensee MDPI, Basel, Switzerland. This article is an open access article distributed under the terms and conditions of the Creative Commons Attribution license (http://creativecommons.org/licenses/by/4.0/). 